\title{
Disección espontánea del tronco celíaco. Una emergencia vascular inusual
}

\author{
Spontaneous dissection of the celiac trunk. An unusual vascular emergency \\ Juan Luis Vélez-Leal' ${ }^{\mathbb{D}}$, Nancy Andrea Pérez-Arroyave ${ }^{1} \mathbb{D}$, Carolina Sánchez-Castro², \\ Luisa María Uribe-Londoño ${ }^{3}$, Jairo Giovanni Moncayo-Viveros ${ }^{4} \mathbb{D}$ \\ 1 Médico, residente de Medicina de urgencias, Universidad CES, Medellín, Colombia. \\ 2 Médica, servicio de Hospitalización, Clínica CES, Medellín, Colombia. \\ 3 Médica, servicio de Urgencias, Clínica CES, Medellín, Colombia. \\ 4 Médico, especialista en Medicina de urgencias, Clínica CES, docente adscrito, Universidad CES, Medellín, Colombia.
}

\section{Resumen}

Introducción. La disección espontánea del tronco o arteria celíaca es una emergencia vascular abdominal inusual, con una incidencia desconocida. Actualmente no existen guías o consensos para su tratamiento, el cual va desde intervenciones vasculares mayores hasta la conducta expectante.

Caso clínico. Paciente masculino de 42 años sin antecedentes patológicos, con dolor abdominal intenso de un mes de evolución. Ante la sospecha clínica de patología inflamatoria se realizó una tomografía computarizada abdominal con medio de contraste, en la que se observó una disección del tronco celíaco asociada a un infarto esplénico. Se realizó manejo endovascular de forma exitosa.

Discusión. La disección espontánea del tronco celíaco es una patología infrecuente, que puede presentarse en pacientes con hipertensión arterial, tabaquismo, enfermedad ateroesclerótica y vasculitis. Los síntomas más comunes son el dolor abdominal asociado a náuseas y vómito. La sospecha clínica debe ser confirmada mediante estudios imagenológicos que orienten al tratamiento conservador, endovascular o quirúrgico, ante la sospecha de infarto intestinal o ruptura arterial.

Conclusiones. La disección espontánea del tronco celíaco es una patología vascular inusual que puede relacionarse con una alta mortalidad. Es importante tener siempre presente la interrelación entre la anatomía vascular abdominal, la anamnesis y el examen físico como elementos claves para realizar este diagnóstico.

Palabras clave: dolor abdominal; arteria celíaca; disección; urgencia; abdominal.

Fecha de recibido: 02/03/2021 - Fecha de aceptación: 26/03/2021 - Publicación en línea: 09/11/2021

Autor de correspondencia: Juan Luis Vélez Leal, Calle 9ª sur \# 29 - 95, edificio Serramonte, apartamento 703, Medellín, Colombia. Teléfono: 31488781 30. Dirección electrónica: velezl.juan@gmail.com

Citar como: Vélez-Leal JL, Pérez-Arroyave NA, Sánchez-Castro C, Uribe-Londoño LM, Moncayo-Viveros JG. Disección espontánea del tronco celiaco. Una emergencia vascular inusual. Rev Colomb Cir. 2022;37:115-21. https://doi.org/10.30944/20117582.908

Este es un artículo de acceso abierto bajo una Licencia Creative Commons - BY-NC-ND https://creativecommons.org/licenses/by-nc-nd/4.0/deed.es 


\begin{abstract}
Introduction. Spontaneous dissection of the celiac artery or trunk is an unusual abdominal vascular emergency, with an unknown incidence. Currently there are no guidelines or consensus for its treatment, which ranges from major vascular interventions to expectant management.

Clinical case. A 42-year-old male patient with no medical history, with intense abdominal pain of one month of evolution. Given the clinical suspicion of inflammatory pathology, an abdominal computed tomography with contrast was performed, in which a dissection of the celiac trunk associated with a splenic infarction was observed. Endovascular management was performed successfully.

Discussion. Spontaneous dissection of the celiac trunk is an infrequent pathology, which can occur in patients with high blood pressure, smoking, atherosclerotic disease, and vasculitis. The most common symptoms are abdominal pain associated with nausea and vomiting. Clinical suspicion must be confirmed by imaging studies that guide conservative, endovascular or surgical treatment in the event of suspected intestinal infarction or arterial rupture.

Conclusions. Spontaneous dissection of the celiac trunk is an unusual vascular pathology that can be associated with high mortality. It is important to always keep in mind the relationship between the abdominal vascular anatomy, anamnesis, and physical examination as key elements in making this diagnosis.
\end{abstract}

Keywords: abdominal pain; celiac artery; dissection; urgency; abdominal.

\section{Introducción}

La disección espontánea de la arteria o tronco celíaco es una patología poco común, con una incidencia desconocida y poco más de un centenar de casos descritos en la literatura médica ${ }^{1-3}$ desde su primera publicación en $1947^{4}$. Sus aspectos epidemiológicos no están claramente establecidos, y no existe un consenso nacional o internacional sobre el manejo de esta entidad ${ }^{2,4-6}$. A continuación, se describe un caso, sus características clínicas, paraclínicas e imagenológicas y se discute el abordaje diagnóstico y terapéutico, de acuerdo con la literatura médica disponible actualmente.

\section{Presentación del caso}

Paciente masculino de 42 años sin antecedentes médicos quien consultó a urgencias por exacerbación de dolor abdominal tipo cólico, de carácter intermitente, localizado en el hipocondrio izquierdo e irradiado al epigastrio e hipocondrio derecho, ocasionalmente asociado a emesis de aspecto bilioso y deposiciones acuosas sin moco ni sangre. No había presentado fiebre, ictericia, coluria ni acolia, sudoración nocturna ni pérdida de peso.
Los signos vitales de ingreso fueron: presión arterial 142/86 mmHg, frecuencia cardiaca 96 latidos por minuto, frecuencia respiratoria 21 por minuto, saturación de oxígeno $98 \%$ al ambiente y temperatura de $36,2^{\circ}$ centígrado. En la valoración inicial se encontró un paciente muy adolorido que había presentado tres episodios de emesis, con dolor a la palpación de flanco izquierdo y epigastrio, pero sin signos de irritación peritoneal. Con sospecha diagnóstica de patología hepatobiliar versus gástrica, se indicó manejo analgésico y se solicitaron los paraclínicos descritos en la tabla 1.

Por la persistencia del dolor se solicitó una tomografía computarizada contrastada de abdomen para descartar patología inflamatoria, encontrando múltiples infartos esplénicos y trastorno de la perfusión hepática, con aparente origen en un defecto de llenado lineal del tronco celíaco (figura 1). De manera consensuada con radiología se solicitó una angiotomografía para esclarecer la lesión vascular, encontrando una disección contenida del tronco celíaco y autolimitada a la arteria hepática común, después del origen de la arteria gástrica izquierda $\mathrm{y}$ antes del origen de la arteria gastroduodenal, sin presencia de aneurismas. No se encontró compromiso isquémico de las vísceras (figura 2). 
Tabla 1. Resultado de los paraclínicos tomados al ingreso del paciente al servicio de urgencias.

\begin{tabular}{lc}
\hline Exámenes de laboratorio & Valor \\
\hline Alanino aminotransferasa & $41 \mathrm{U} / \mathrm{ml}$ \\
Aspartato aminotransferasa & $26,3 \mathrm{U} / \mathrm{ml}$ \\
Fosfatasa alcalina & $75 \mathrm{U} / \mathrm{ml}$ \\
Bilirrubina total/directa & $0,28 \mathrm{mg} / \mathrm{dL} / 0,13 \mathrm{mg} / \mathrm{dL}$ \\
Lipasa & $27,5 \mathrm{U} / \mathrm{L}$ \\
Creatinina & $1,08 \mathrm{mg} / \mathrm{dL}$ \\
Nitrógeno ureico en sangre & $13,59 \mathrm{mg} / \mathrm{dL}$ \\
Serie roja & $15.800 \mathrm{~mm} 3$ \\
Serie blanca & $8120 \mathrm{~mm} 3$ \\
Tiempo parcial de tromboplastina & $24,7 \mathrm{segundos}$ \\
Tiempo de protrombina & $11 \mathrm{segundos}$ \\
International normalized ratio (INR) & $1,06 \mathrm{segundos}$ \\
\hline Gases arteriales & \\
\hline pH & 7,513 \\
Presión arterial de oxígeno & $77,9 \mathrm{~mm} / \mathrm{Hg}$ \\
Presión arterial de dióxido de carbono & $21 \mathrm{~mm} / \mathrm{Hg}$ \\
Bicarbonato arterial & $16,6 \mathrm{mmol} / \mathrm{L}$ \\
Exceso de base & $-6.6 \mathrm{mmol} / \mathrm{L}$ \\
Saturación arterial de oxígeno & $97 \%$ \\
Lactato & $3,64 \mathrm{mmol} / \mathrm{L}$ \\
\hline
\end{tabular}

Luego de la valoración por los grupos de cirugía vascular y hemodinamia, se inició antiagregación con ácido acetil salicílico (ASA), control de la presión arterial, analgesia multimodal, hidratación endovenosa y reposo intestinal, sin requerir medicamentos vasoactivos por estar hemodinámicamente normal.

Debido a la persistencia del dolor abdominal con episodios de gran intensidad, asociados a emesis, con alta sospecha de isquemia visceral en curso, se decidió realizar tratamiento endovascular, encontrando disección del tronco celíaco en su ostium y tercio proximal, el cual causaba una oclusión parcial y bajo flujo en la arteria hepática, con ausencia de flujo en la arteria gastroduodenal. Se practicó una angioplastia con balón e implantación de una prótesis de 7 x 27 mm, mejorando el flujo de los vasos distales, sin estenosis residual y sin complicaciones asociadas.
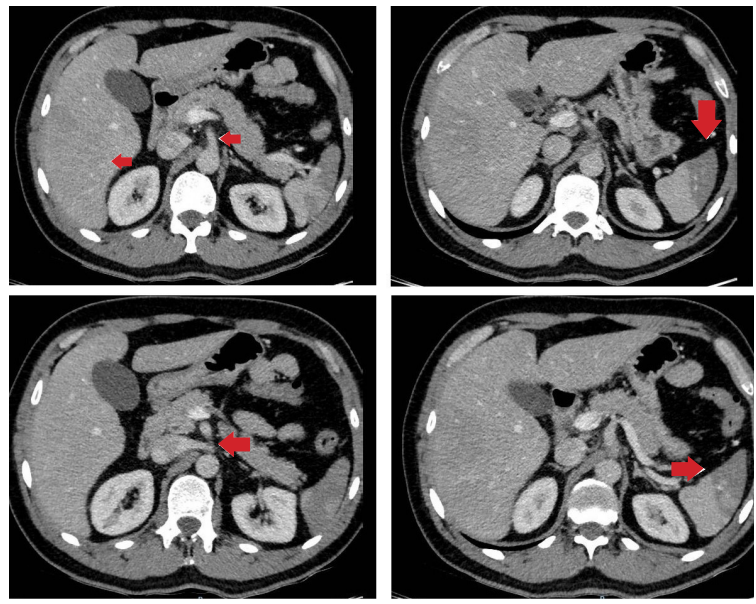

Figura 1. Tomografía computarizada abdominal con contraste venoso. Se observan múltiples infartos esplénicos y trastorno de la perfusión hepática que tienen aparente origen en un defecto de llenado lineal del tronco celíaco (flechas).
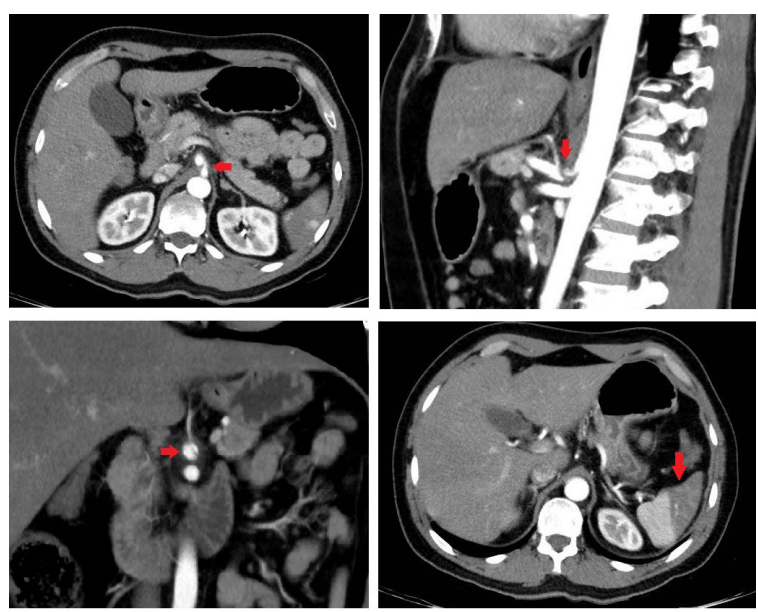

Figura 2. Disección contenida del tronco celíaco autolimitada en la arteria hepática común después del origen de la arteria gástrica izquierda y antes del origen de la arteria gastroduodenal (flecha roja). No se observan aneurismas o placas ateromatosas, se observan áreas de infarto esplénico.

El paciente continuó observación y monitorización en el servicio de urgencias con una evolución favorable dada por la mejoría total del dolor abdominal, desaparición de los episodios eméticos y adecuada tolerancia de la vía oral. Aproximadamente a las 24 horas de su ingreso fue dado de alta con prescripción de ASA de forma 
indefinida y control ambulatorio por cirugía vascular y hemodinamia. Durante los controles por consulta externa el paciente permaneció asintomático.

\section{Discusión}

Cerca del 4 - $5 \%$ de todos los pacientes que consultan a un servicio de urgencias lo hacen por algún tipo de dolor abdominal, representando el motivo de consulta más frecuente en los servicios de emergencias generales en todo el mundo ${ }^{7}$. El dolor abdominal inespecífico constituye uno de los grandes retos clínicos de la medicina. Si bien puede involucrar numerosos procesos patológicos, las emergencias vasculares intraabdominales pueden ser la causa, con un alto potencial de mortalidad, por lo que siempre se deben sospechar, aunque el examen físico no arroje mayor información diagnóstica ${ }^{8}$.

Para comprender la relevancia clínica del compromiso del tronco celiaco, es necesario recordar brevemente la anatomía vascular intrabdominal. La aorta da lugar a múltiples vasos pares e impares dentro del abdomen, las arterias renales, suprarrenales y gonadales son pares e irrigan dichas estructuras, mientras la arteria celiaca, la mesentérica superior y la inferior son impares e irrigan la mayoría del tracto gastrointestinal. El tronco celíaco se origina directamente de la aorta en ángulo de aproximadamente $90^{\circ} \mathrm{y}$ de él emergen tres ramas: la arteria esplénica, la gástrica izquierda y la hepática común, las cuales irrigan las estructuras desde el esófago distal hasta la segunda porción del duodeno, el bazo, el hígado y parte del páncreas ${ }^{9}$.

La disección de estas arterias es rara, siendo la del tronco celíaco la más infrecuente ${ }^{10}$.

En una revisión sistemática de series de casos de disección espontánea del tronco o arteria celíaca publicados entre 1987 y 2015, se documentó que el $85,1 \%$ de los pacientes eran de sexo masculino, con una edad media entre $53,1 \pm 9,3$ años ${ }^{11}$. Los factores de riesgo más frecuentemente encontrados son hipertensión arterial en un $31 \%$ y tabaquismo en un $23 \%{ }^{2,10,11}$; otros menos comunes son enfermedad ateroesclerótica y vasculitis ${ }^{1}$. El $99 \%$ de los pacientes al ingreso pueden presentar dolor abdominal y un $14,8 \%$ náuseas y vómito ${ }^{1,11}$. Los pacientes con sangrado agudo e isquemia hepática presentan el peor desenlace, con una mortalidad cercana al $40 \%{ }^{11}$.

Las características demográficas y los síntomas de nuestro caso presentado concuerdan en parte con lo informado en la literatura, aunque el paciente no presentaba los factores de riesgo comúnmente asociados. Llama la atención la particularidad de las características del dolor descritas por el paciente en cuanto al tiempo de evolución de un mes, su localización en el epigástrico con irradiación a ambos hipocondrios y las varias consultas médicas previas, sin haber logrado un diagnóstico etiológico.

La fisiopatología de esta enfermedad se corresponde con el compromiso de la integridad del flujo sanguíneo visceral, el cual puede afectarse tanto por eventos isquémicos como hemorrágicos. Los eventos embólicos en el tronco celíaco son menos comunes que en las arterias mesentéricas, debido a su angulación al emerger de la aorta ${ }^{9}$. La formación de los aneurismas abdominales está en relación con los factores de riesgo cardiovasculares ampliamente descritos y su principal complicación la constituye la ruptura, relacionada de forma directa con el tamaño de la dilatación. La disección, mucho menos frecuente, afecta la perfusión distal debido a que la falsa luz de la zona disecada compromete el flujo sanguíneo hacia los órganos blanco 9,12.

En cuanto al dolor abdominal de origen vascular, existen entidades que obligan a tener un diagnóstico certero lo más pronto posible, debido a su progresión que depende del tiempo de evolución. Dichas situaciones deben sospecharse ante la presencia de hipotensión, alteración del estado de conciencia, dolor intenso y acidosis metabólica, enfatizando que diversas patologías potencialmente mortales suelen no tener hallazgos de gran valor diagnóstico al examen físico ${ }^{12,13}$. Por lo anterior, las imágenes diagnósticas, especialmente la tomografía computarizada de abdomen total con uso de contraste intravenoso, se ha convertido en la herramienta diagnóstica de preferencia 
para múltiples escenarios de dolor abdominal no asociado a trauma, dado que su rendimiento en cuanto a sensibilidad y especificidad es satisfactorio en la mayoría de diagnósticos críticos ${ }^{7,13}$.

Con respecto a los trastornos vasculares, y en particular ante la sospecha de disección espontánea del tronco celíaco, la tomografía computarizada con técnica de reconstrucción angiográfica es el método de elección, ya que puede demostrar una imagen con doble lumen, y además permite determinar el tamaño, localización y otros hallazgos intrabdominales asociados, como hemorragia o necrosis ${ }^{3,14}$. La amplia disponibilidad de la angiotomografía, los tiempos de adquisición más cortos y la reconstrucción tridimensional mejorada, la convierten en una imagen diagnóstica ideal, sin embargo, la disección de la artería celíaca también se puede observar en la angiografía por resonancia magnética, ecografía Dúplex y angiografía convencional ${ }^{15,16}$.

Los hallazgos imagenológicos incluyen la presencia de un colgajo en la íntima, un trombo mural o periférico en la luz del tronco celíaco, infiltración grasa y aneurismas ${ }^{10,15,17}$. Es posible que el colgajo de la íntima pase desapercibido, por lo que la presencia de un trombo mural no solo debe guiar el diagnóstico, sino también constituir un factor diferencial de la trombosis celíaca, que requiere un tratamiento diferente ${ }^{16}$. La presencia de aneurismas se relaciona especialmente con la ateroesclerosis y es raro que se presente como una complicación aislada de la disección de la arteria celíaca ${ }^{17}$.

La disección puede extenderse hacia los vasos adyacentes, en cuyo caso podrían observarse aneurismas, ausencia de flujo e infartos viscerales, tanto en vísceras huecas como sólidas ${ }^{17}$, lo que significa que las emergencias vasculares intraabdominales son patologías tiempo-dependientes, que al comprometer la viabilidad de los órganos intraabdominales favorecen el infarto, la translocación bacteriana y, eventualmente, pueden llevar a la muerte por sepsis de origen abdominal ${ }^{9}$.

El objetivo del tratamiento de esta patología es reducir o eliminar la falsa luz, prevenir la ruptura de la disección y corregir el defecto en la arteria ${ }^{3,6}$. En la revisión sistemática publicada por Cavalcante et al., el manejo fue conservador en un 79,1\%, endovascular en un $11,8 \%$ y quirúrgico en un $8,9 \%$ de los casos. Se ha reportado que, sin importar la morfología de la disección, la mayoría de los casos se manejan de forma conservadora con tratamiento antihipertensivo, con o sin terapia anticoagulante y antiagregante ${ }^{4,18-20}$. La terapia anticoagulante se fundamenta en el alto riesgo de trombosis de las arterias viscerales dado su menor diámetro y flujo más lento en comparación con la aorta ${ }^{21}$. Sin embargo, hay reportes de manejo expectante exitoso en pacientes asintomáticos en quienes el hallazgo fue incidental ${ }^{19}$.

En pacientes con casos leve o moderadamente sintomáticos, se ha descrito anticoagulación por un mínimo de 3 a 6 meses, como prevención de complicaciones tromboembólicas, lo anterior aunado al control estricto de la presión arterial ${ }^{4,6}$. Si bien la meta del manejo médico es evitar la progresión de la disección y las complicaciones tromboembólicas ${ }^{18}$, se ha descrito que hasta un tercio de los pacientes requieren posteriormente algún tipo de intervención ${ }^{21}$. La literatura describe que, si se prevé anticoagulación por más de seis meses o si persiste el dolor o los síntomas y signos de isquemia intestinal o visceral progresiva, estarán indicadas medidas intervencionistas como manejo endovascular con prótesis y revascularización quirúrgica mediante resección del segmento disecado y anastomosis o baipás, e implantación de prótesis endovasculares en la arteria comprometida ${ }^{4-6,18,20-21}$. En el caso presentado, ante la sospecha clínica y paraclínica de isquemia en curso, se optó por manejo endovascular obteniendo un resultado exitoso, logrando un flujo arterial satisfactorio y la desaparición del dolor luego del implante de la prótesis. Este caso se asemeja a lo reportado en el estudio de Wang ${ }^{6}$.

Las indicaciones absolutas de manejo invasivo o de cirugía de emergencia incluyen la sospecha de infarto intestinal o ruptura arterial ${ }^{21}$. En caso de intervención quirúrgica, es recomendable realizar estudio histopatológico de la arteria resecada pues ante el hallazgo de vasculitis se debe 
incluir antiinflamatorios y corticoesteroides en el manejo médico ${ }^{4}$. La tendencia actual corresponde al manejo mínimamente invasivo siempre y cuando la anatomía sea favorable y se cuente con la experiencia en el centro de atención ${ }^{18}$. Para los pacientes tratados de forma conservadora se aconseja el seguimiento imagenológico ${ }^{21}$.

\section{Conclusiones}

El conocimiento de esta patología por parte de los médicos generales, especialistas en urgencias y cirujanos que atienden pacientes con patologías agudas, es de suma importancia. Aunque se trata de casos inusuales, el conocimiento de la anatomía vascular abdominal y de las claves diagnósticas mediante la anamnesis y el examen físico, deben hacer sospechar esta alteración intraabdominal, relacionada con una alta morbi-mortalidad.

El personal médico debe tener conocimiento acerca de las ayudas diagnósticas a utilizar cuando se sospechan patologías críticas, asegurando un diagnóstico oportuno y su manejo consecuente.

\section{Cumplimiento de normas éticas}

Consentimiento informado: Este artículo es una revisión de la literatura y la descripción retrospectiva de un caso clínico y no implicó riesgo alguno para el paciente, por lo que no se requiere consentimiento informado.

Conflicto de interés: Ninguno declarado por los autores.

Fuente de financiación: Financiado con recursos propios de los autores.

\section{Contribución de los autores}

Concepción y diseño del estudio: Juan Luis Vélez-Leal, Nancy Andrea Pérez-Arroyave.

Adquisición de datos: Juan Luis Vélez-Leal, Nancy Andrea Pérez-Arroyave

Análisis e interpretación de datos: Juan Luis Vélez-Leal, Nancy Andrea Pérez-Arroyave, Carolina Sánchez-Castro.

Redacción del manuscrito: Juan Luis Vélez-Leal, Nancy Andrea Pérez-Arroyave, Carolina Sánchez-Castro, Luisa María Uribe-Londoño, Jairo Giovanni Moncayo-Viveros.

Revisión crítica: Juan Luis Vélez-Leal, Nancy Andrea Pérez-Arroyave, Carolina Sánchez-Castro, Luisa María Uribe-Londoño, Jairo Giovanni Moncayo-Viveros.

\section{Referencias}

1. Galastri FL, Cavalcante RN, Motta-Leal-Filho JM, De Fina B, Affonso BB, de Amorim JE, et al. Evaluation and management of symptomatic isolated spontaneous celiac trunk dissection. Vasc Med. 2015;20:358-63. https://doi.org/10.1177/1358863X15581447

2. Dimusto PD, Oberdoerster MM, Criado E. Isolated celiac artery dissection. J Vasc Surg. 2015;61:972-6. https://doi.org/10.1016/j.jvs.2014.10.108

3. Esteban-Zubero E, Flamarique-Pascual Á, Zubero EE. Disección aneurismática del tronco celíaco, una entidad infrecuente. Iatreia. 2017;30:194-8. https://doi.org/10.17533/udea.iatreia.v30n2a08

4. Abugroun A, Natarajan A, Daoud H, Khalaf H. Spontaneous celiac artery dissection presenting with splenic infarction: a case report. Gastroenterol Res. 2018;11:379-82. https://doi.org/10.14740/gr1065w

5. Endler GT, Curfman KR, Hwang JH, Gray JL. A complicated entity: acute celiac artery dissection with resultant pancreatitis, duodenitis, and cholecystitis. Case Rep Vasc Med. 2020;20:1-5. https://doi.org/10.1155/2020/8453168

6. Wang HT, Yu ZH, Tu C, Lu B. Interventional treatment of isolated dissection of the celiac artery: A case report and literature review. Medicine. 2018;97:95-7. https://doi.org/10.1097/MD.0000000000011026

7. Gangadhar K, Kielar A, Dighe MK, O'Malley R, Wang C, Gross JA, et al. Multimodality approach for imaging of non-traumatic acute abdominal emergencies. Abdom Radiol. 2016;41:136-48. https://doi.org/10.1007/s00261-015-0586-6

8. Macaluso CR, McNamara RM. Evaluation and management of acute abdominal pain in the emergency department. Int J Gen Med. 2012;5:789-97. https://doi.org/10.2147/IJGM.S25936

9. Lewiss RE, Egan DJ, Shreves A. Vascular abdominal emergencies. Emerg Med Clin North Am. 2011;29:25372. https://doi.org/10.1016/j.emc.2011.02.001

10. Ferguson C, Rockley M, Panu A, Turnbull R. A unique case of isolated, spontaneous, symptomatic celiac trunk dissection. SAGE Open Med Case Reports. 2015;3:2050313. https://doi.org/10.1177/2050313X14565423

11. Cavalcante RN, Motta-Leal-Filho JM, De Fina B, Galastri FL, Affonso BB, de Amorim JE, et al. Systematic literature review on evaluation and management of isolated spontaneous celiac trunk dissection. Ann Vasc Surg. 2016;34:274-79. https://doi.org/10.1016/j.avsg.2015.12.009

12. Lech C, Swaminathan A. Abdominal aortic emergencies. Emerg Med Clin North Am. 2017;35:847-67. https://doi.org/10.1016/j.emc.2017.07.003

13. Del Arco-Galán C, Parra-Gordo ML, Garcia-Casasola-Sanchez G. Imaging tests in acute abdominal pain. Rev Clin Esp. 2008;208:520-4. https://doi.org/10.1157/13128678 
14. Sakamoto I, Ogawa Y, Sueyoshi E, Fukui K, Murakami $\mathrm{T}$, Uetani M. Imaging appearances and management of isolated spontaneous dissection of the superior mesenteric artery. Eur J Radiol. 2007;64:103-10. https://doi.org/10.1016/j.ejrad.2007.05.027

15. Vaidya S, Dighe M. Spontaneous celiac artery dissection and its management. J Radiol Case Rep. 2010;4:30-3. https://doi.org/10.3941/jrcr.v4i4.408

16. Kim JH, Roh BS, Lee YH, Choi SS, So BJ. Isolated spontaneous dissection of the superior mesenteric artery: percutaneous stent placement in two patients. Korean J Radiol. 2004;5:134-8. https://doi.org/10.3348/kjr.2004.5.2.134

17. D’Ambrosio N, Friedman B, Siegel D, Katz D, Newatia A, Hines J. Spontaneous isolated dissection of the celiac artery: CT findings in adults. Am J Roentgenol. 2007;188:506-11.

https://doi.org/10.2214/AJR.06.0315
18. Hoglund JR, Blackwell JH, Gibbs MA. Spontaneous celiac artery dissection. Am J Emerg Med. 2020;38:1545.e31545.e5. https://doi.org/10.1016/j.ajem.2020.04.041

19. Duraikannu C, Karunakaran P, Haithrous SA, Pulupula VNK. Natural course of incidentally detected isolated Celiac Artery Dissection with hepatic artery occlusion. Radiol Case Reports. 2020;15:479-83.

https://doi.org/10.1016/j.radcr.2020.01.028

20. Lee SI, Kim JJ, Yang HJ, Lee K. Spontaneous dissection of celiac trunk with concurrent splenic artery dissection. Clin Exp Emerg Med. 2015;2:256-9.

https://doi.org/10.15441/ceem.15.030

21. Hedfi M, Messaoudi Y, Chouchene A. Conservative management of isolated superior mesenteric artery and celiac trunk dissection: A case report and literature review. J Clin Diagnostic Res. 2016;10:24-6. https://doi.org/10.7860/JCDR/2016/24177.8877 